\title{
Particle image velocimetry measurement of turbulent wake past circular cylinder at Reynolds numbers from $2.5 \cdot 10^{3}$ to $1.5 \cdot 10^{4}$
}

\author{
Ondřej Sterly ${ }^{1 *}$ \\ ${ }^{1}$ University of West Bohemia in Pilsen, Univerzitní 22, Pilsen, Czech Republic
}

\begin{abstract}
A canonical case of air flow past a circular cylinder is studied by using Particle Image Velocimetry technique. This contribution focus to the ensemble statistics (first and second moment) of the stream-wise and transverse velocity component as well as to the in-plane vorticity component. Although the range of explored Reynolds numbers is narrow, we observe a significant shortening of recirculation bubble within this range.
\end{abstract}

\section{Introduction}

Many of engineering applications have to deal with interaction of flow and a solid structure [1]. This includes the effectivity of transportation devices, resistance of buildings against weather or the power generation. The last means not only the complex flow inside a closed steam turbine [2,3] or the open wind turbines [4], but also the heat exchanger flows as well as the natural convective cooling $[5,6,7]$. Lot of experiments focusing this question is performed with cryogenic helium [8] due to its high thermal expansivity and thus the possibility of reach high Grashof numbers (in the relevant literature, the Rayleigh number is often used instead [7]). The experimental study of turbulent flows has a very wide practical uses.

The effect of an obstacle to the flow can be mainly studied within the wake [9], i.e. the part of fluid affected by the former interaction with the obstacle and carried downstream with the convective velocity component. The wake is typical by periodic oscillations [10] not only, if the obstacle motion is periodic $[11,12]$. The most studied case of wake flow is the wake past a prismatic circular cylinder with axis perpendicular to the incoming flow $[9,36]$, which is the case studied in this contribution as well. This setup is very simplified and for a practical purposes a more complex prismatic obstacle (or profile) is used [13, 14]. Of course, a nonprismatic obstacles are studied as well $[15,16]$, but this approach usually needs a more complex Stereo Particle Image Velocimetry technique [17, 18], while here we use this method only in its 2D configuration, which still offers a deep insight into the physics of the flow [19].

Despite its simplicity, the experimental configuration of a prismatic cylinder placed perpendicularly to the incoming flow shows a lot of interesting physical phenomena, e. g. the flow is three dimensional, although the geometry has two-dimensional symmetry [20], the

\footnotetext{
* Corresponding author: sterlyo@students.zcu.cz
} 
flow is periodic even in a steady incoming flow [10]. Therefore, new ideas are often tested in this simple case [21,22]. One of the most fascinating properties of this simple canonical experiment is the wealth of regimes depending only on a single parameter: Reynolds number. Figure 1 shows the variation of base pressure coefficient $C_{p b}$ (i.e. non-dimensionalized static pressure $p_{b}$ at the trailing point of the body) with Reynolds number spanning 6 orders of magnitude as it is published by Williamson [23].

$$
C_{p b}=\frac{2\left(p_{b}-p_{\infty}\right)}{\rho U_{r e f}^{2}}
$$

Where $\rho$ is the fluid density, $U_{\text {ref }}$ is the reference velocity and $p_{\infty}$ is the static pressure far enough (at the wind tunnel wall)

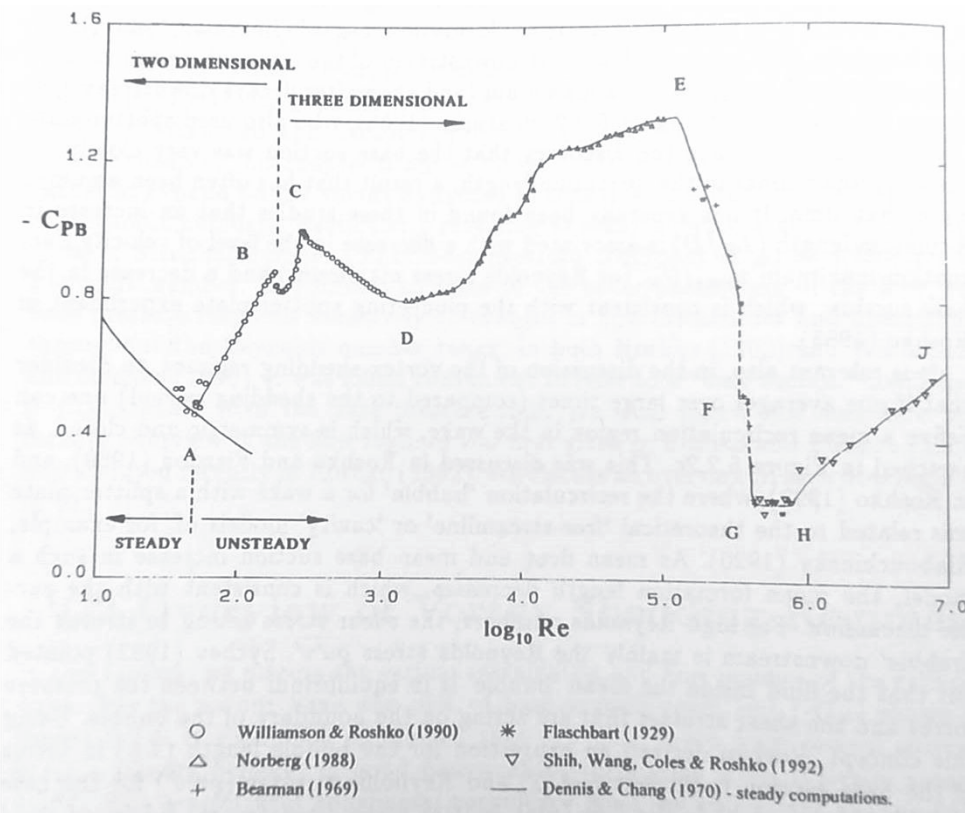

Fig. 1. Regimes of the wake past a circular cylinder shown in the terms of base pressure coefficient dependence on Reynolds number. The figure is copied from the book chapter [23] by C. H. K. Williamson. The mentioned works are references [24 - 28] except for Flaschbart (1929), which I did not find. The regimes distinguished by Williamson are denoted A - J. C Kluwer Academic Publishers.

The regimes observed in the base pressure development in Figure 1 are described in more details by Williamson [23]. The regime up to $\mathrm{A}$ is a laminar steady regime, the laminar vortex shedding is observed between $\mathrm{A}$ and $\mathrm{B}$. Between $\mathrm{B}$ and $\mathrm{C}$, there is a transition to threedimensional wake. Up to $\mathrm{D}$, the fine-scale three-dimensionalities increase their disorder. The steep decrease from $\mathrm{E}$ to $\mathrm{G}$ is caused by the asymmetric reattachment of the bubble (sometimes called critical transition). Between $\mathrm{G}$ and $\mathrm{H}$, the reattachment become symmetrical and, finally, beyond $\mathrm{H}$ the boundary layer transits. More details can be found in the book chapter [23].

The present contribution shows results of a student project of measuring the wake past a circular cylinder by using Particle Image Velocimetry technique at intermediate Reynolds numbers from $2.5 \cdot 10^{3}$ to $1.5 \cdot 10^{4}$, which is very narrow range in comparison with the data in literature. 


\subsection{Experimental setup}

The measurement has been performed inside the open wind tunnel at University of West Bohemia in Pilsen [29, 30]. The commercial Particle Image Velocimetry (PIV) system from company Dantec consists of a 4 MPix FlowSense MkII camera and a solid-state double pulse laser New Wave Solo of dominant wavelength $532 \mathrm{~nm}$ and pulse energy up to $500 \mathrm{~mJ}$. The used system is the "slow" one with maximal repeating frequency $7.4 \mathrm{~Hz}$, therefore it is not possible to measure the time development of individual vortical structures in the wake. The used lens of focal distance $60 \mathrm{~mm}$ together with the used camera gives the spatial resolution of acquired images $34 \times 34 \mathrm{~mm}$, i.e. $3.4 \times 3.4$ the cylinder diameter.

This PIV system has been used for many measurements in the past, for example the flow inside an axial single stage-turbine [3, 31], which was not so easy as it sounds [32]. From the laboratory experiments, this system participated in the measurement of secondary flow in a corner of channel [33], measurement of turbulent mixing past a grid [34] and past a strangeshaped counter-swirler [16] or in a turbulent jet [35].

The cylinder is made of aluminum, its diameter is $10 \mathrm{~mm}$ and height $125 \mathrm{~mm}$ filling the entire height of the wind tunnel test section. The Reynolds number has been controlled via velocity ranging from 3.8 to $23.0 \mathrm{~m} / \mathrm{s}$, which differs only 6 times, but still this range displays an interesting phenomena of shortening of the recirculation bubble. The working fluid is atmospheric air of temperature $19.1 \pm 1.3{ }^{\circ} \mathrm{C}$. The seeding particles are produced by a commercial fog generator Safex.

In the following, the stream-wise velocity is denoted $u$, the transverse velocity component is denoted $v$. The third velocity component along the cylinder axis is not measured in current configuration. The ensemble consists of around 700 snapshots. The coordinate system displayed in figures is in millimeters and it originates at the beginning of the field of view (FoV), the position of the cylinder is $[-5,16]$ in the coordinate system of the FoV, the cylinder radius is $5 \mathrm{~mm}$, its height is $125 \mathrm{~mm}$ filling the entire height of the wind tunnel test section.

\section{Results}

The focus is given to the ensemble averaged quantities, the stream-wise velocity component $u$ and the span-wise velocity component $v$. The ensemble average $u$ of instantaneous velocity signal $u_{i}$ is calculated as

$$
u(\vec{x})=\left\langle u_{i}(\vec{x})\right\rangle=\frac{1}{N} \sum u_{i}(\vec{x})
$$

And for the second velocity component $v$ analogically. Here, $\vec{x}$ is any spatial position within the field of view, $N$ is the number of snapshots in the ensemble, i.e. around 700 in this measurement.

Figure 2a displays the evolution of the ensemble averaged stream-wise velocity $u$ along the wake axis. The area of negative velocity called recirculation bubble is formed past the body and with increasing Reynolds number it shortens significantly with length around 2.3 cylinder diameters at the lowest velocity down to 0.9 diameters at the highest velocity. Accordingly, the position of minimal velocity moves as well. The deep of the minimal velocity first slightly decreases down to -0.30 times reference velocity at $\mathrm{Re}=5.1 \cdot 10^{4}$ followed by slight increase to values around -0.25 times reference velocity at the higher Reynolds numbers. 

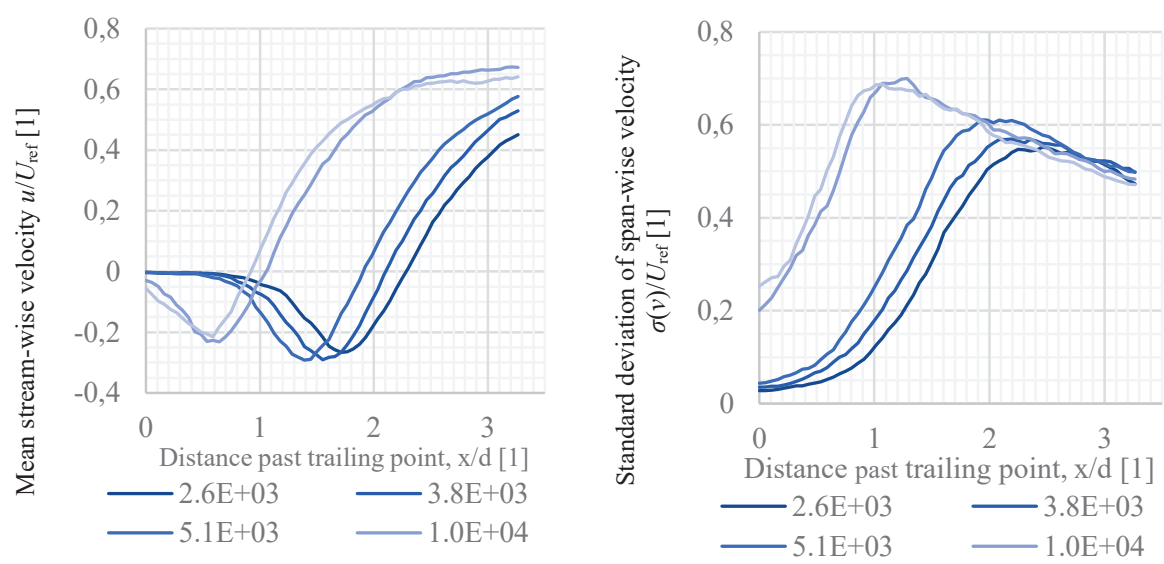

Fig. 2. (a) Ensemble average of stream-wise velocity component $u$ as a function of distance past trailing point of the cylinder, the profile is taken at the wake axis. (b) Standard deviation of transverse velocity component $v$ as a function of distance past trailing point. The number in the legend represents the Reynolds number with the notation that "E+n" plays for " $10^{\mathrm{n}}$ ", we apologize for this inconvenient notation.
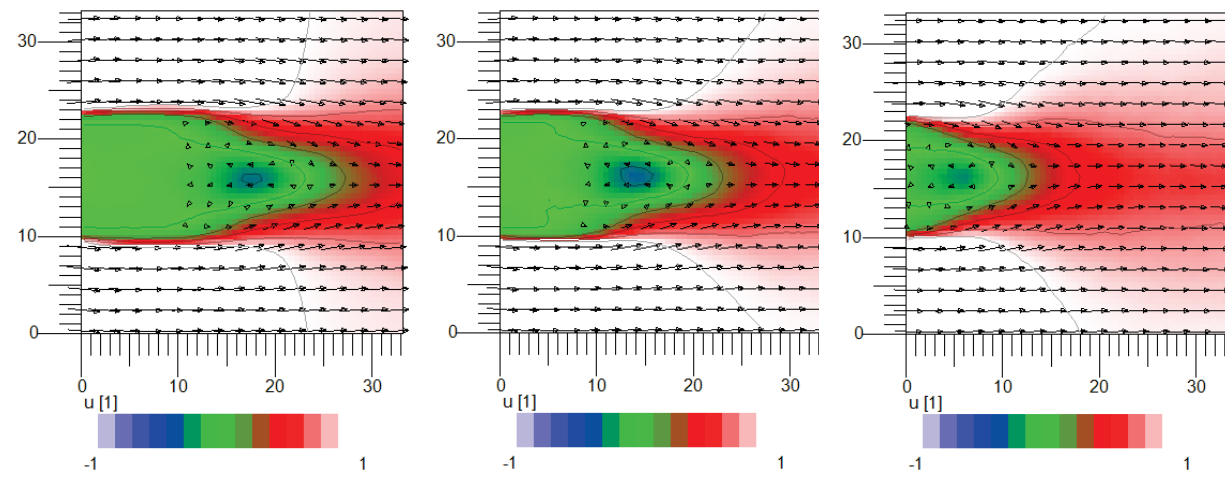

Fig. 3. Ensemble average of measured stream-wise velocity component $u$. The Reynolds numbers are from left: $2.55 \cdot 10^{3}, 5.1 \cdot 10^{3}$ and $1.53 \cdot 10^{4}$. The coordinates are in millimeters, horizontal one represents stream-wise direction, the vertical one the transverse direction. The cylinder axis is perpendicular to the field of view and it is placed left hand side of it in a such way, that the trailing point has coordinates $[0,16] \mathrm{mm}$.

Figure 3 shows the spatial distribution of the ensemble averaged stream-wise velocity and Fig. 4 displays its standard deviation, i.e. second raw moment, which is calculated using:

$$
\sigma[u](\vec{x})=\sqrt{\left\langle\left(u_{i}(\vec{x})-\langle u\rangle(\vec{x})\right)^{2}\right\rangle}
$$

Symbol $\langle\cdots\rangle$ means the average, $u_{i}$ denotes the instantaneous velocity at certain position $\vec{x}$. The spatial distribution of $\sigma[u]$ differs significantly from that of $\sigma[v]$ displaying a pair of peaks past each side of the cylinder. Those peaks are displayed in Fig. 5. First, the peaks are sharp and probably much higher than the value measured by finite resolution of PIV system. Later downstream a sudden widening follows splashing the fluctuating fluid mainly towards the wake axis, later dissipating outside of the wake as well. This is in contrast to the 
distribution of fluctuations in transverse component $v$, Fig. 7, which has a maximum in between the peaks of stream-wise velocity fluctuations.
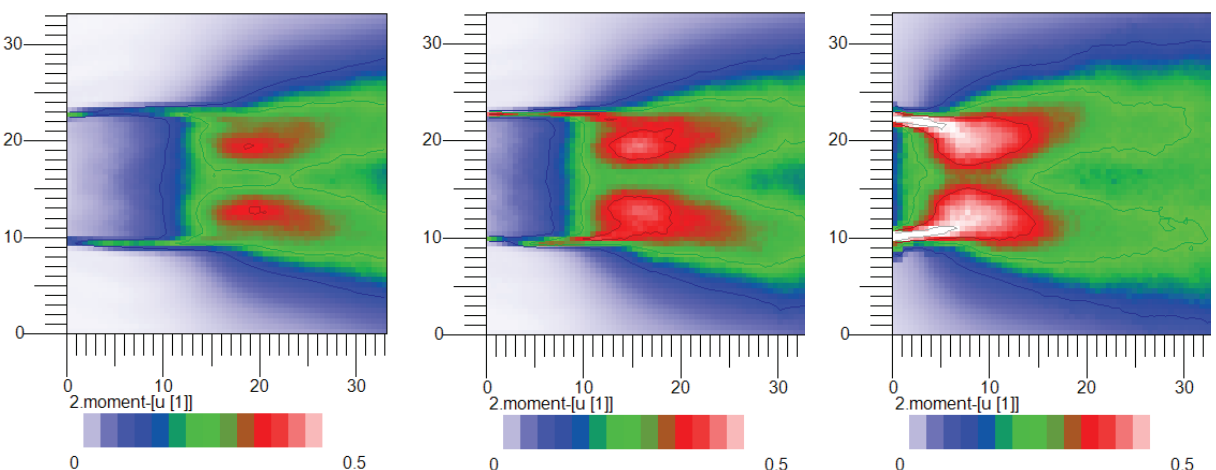

Fig. 4. Standard deviation of measured stream-wise velocity component $u$. The Reynolds numbers are from left: $2.55 \cdot 10^{3}, 5.1 \cdot 10^{3}$ and $1.53 \cdot 10^{4}$.
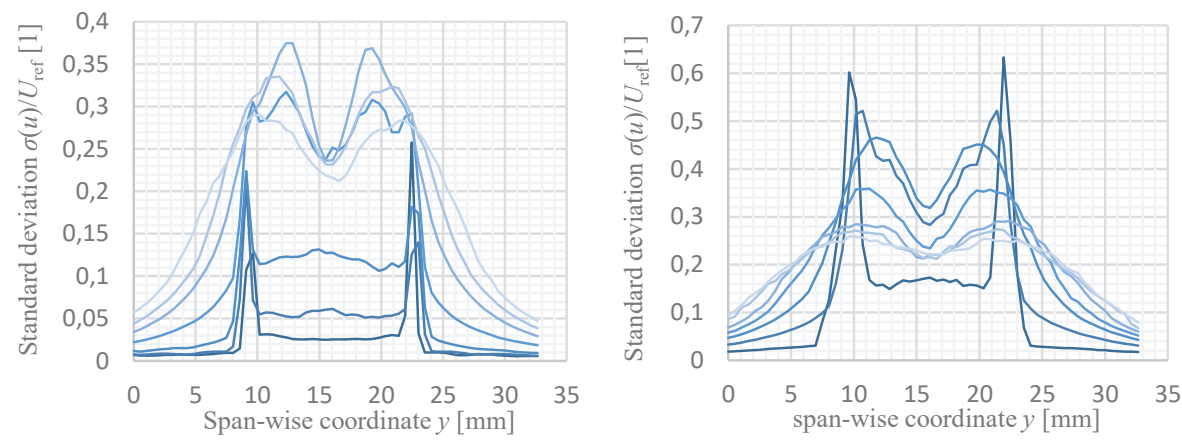

Fig. 5. Graphs showing cuts through the ensemble of standard deviations of the velocity component $u$. Darker color means lesser distance from measured cylinder. Values of second moment are on the $y$ axis, on the $\mathrm{x}$ axis are $\mathrm{y}$ coordinates. The Reynolds numbers are from left: $2.55 \cdot 10^{3}$ and $1.53 \cdot 10^{4}$.
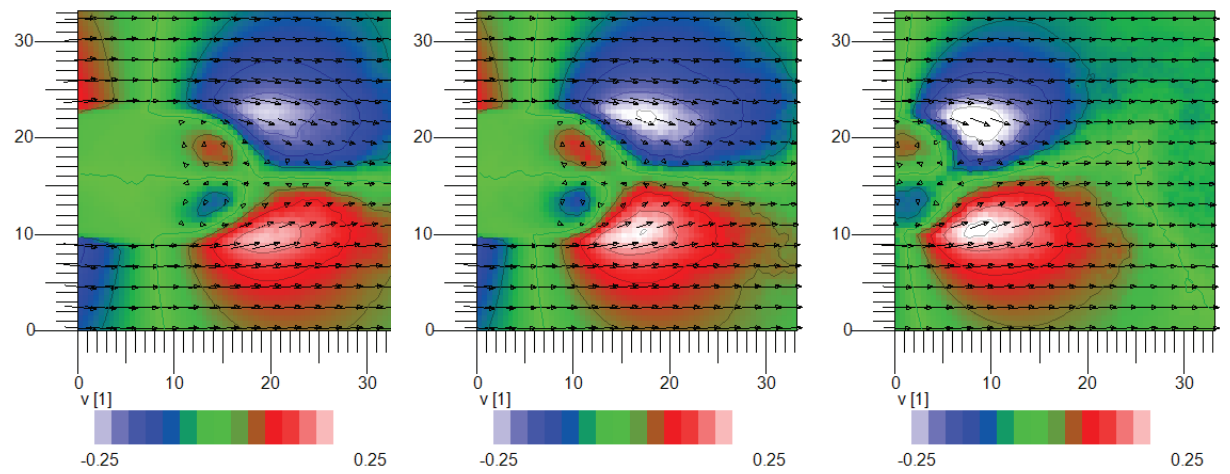

Fig. 6. Ensemble average of measured stream-wise velocity component $\mathrm{v}$. The Reynolds numbers are from left: $2.55 \cdot 10^{3}, 5.1 \cdot 10^{3}$ and $1.53 \cdot 10^{4}$. 

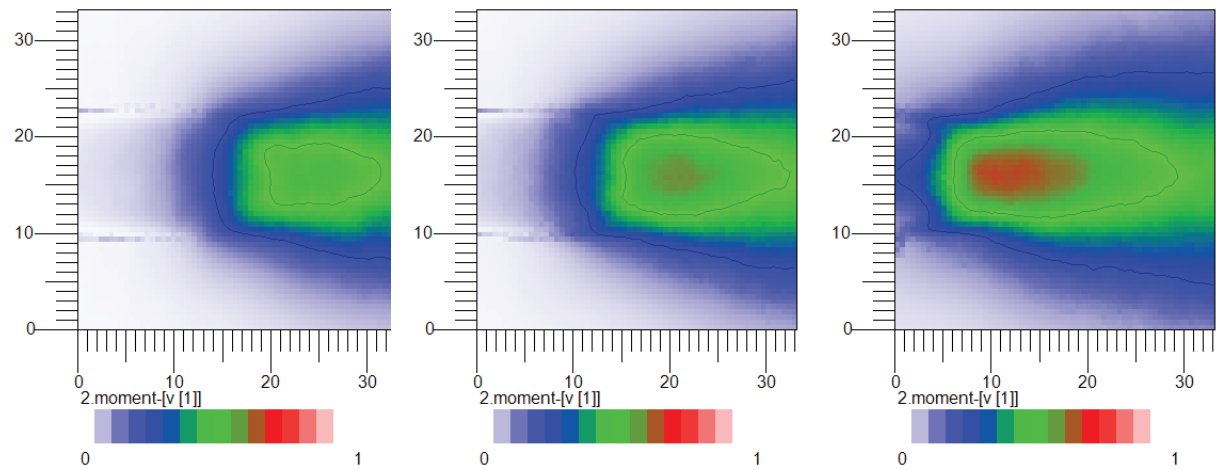

Fig. 7. Standard deviation of measured span-wise velocity component $v$. The Reynolds numbers are from left: $2.55 \cdot 10^{3}, 5.1 \cdot 10^{3}$ and $1.53 \cdot 10^{4}$.
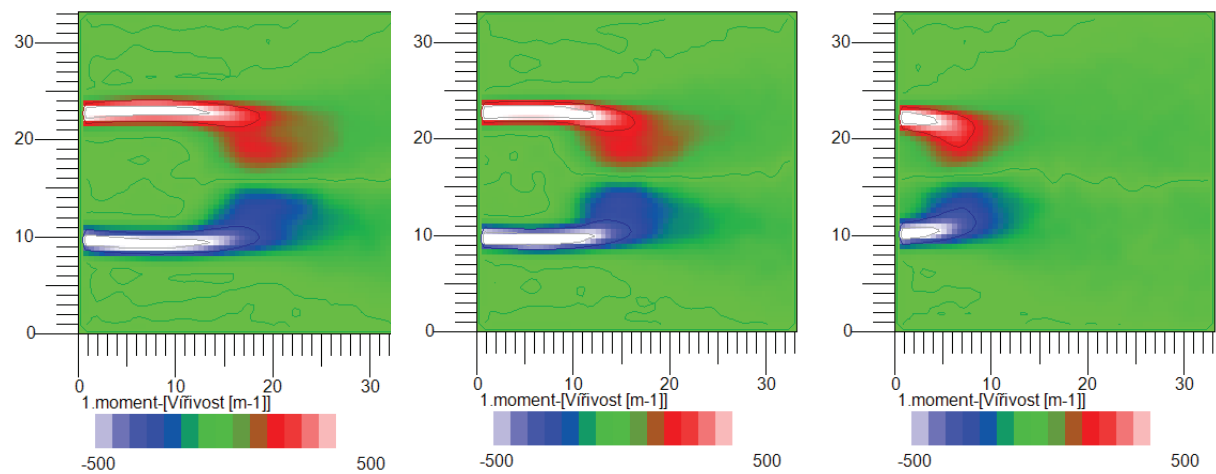

Fig. 8. Mean vorticity component parallel with the cylinder axis. The velocity field is normalized by the reference velocity $U_{\text {ref }}$ in order to compare distributions at different velocities and thus the effective unit of vorticity changes from $\left[\mathrm{s}^{-1}\right]$ to $\left[\mathrm{m}^{-1}\right]$. The Reynolds numbers are from left: $2.55 \cdot 10^{3}$, $5.1 \cdot 10^{3}$ and $1.53 \cdot 10^{4}$.

Vorticity is calculated using rotation of velocity as:

$$
\vec{\omega}=\nabla \times \vec{u}
$$

Note that only the component perpendicular to the field of view can be calculated as only two velocity components are measured. Thus this quantity behaves as a scalar in the data. The shear layers are characterized by strong vorticity signal, see Fig. 8, while around the stagnation point it disappears, which does not mean, that the fluid was quiet there, the opposite is true, as can be visible in the map of standard deviation (second raw moment) of vorticity in Fig. 9. 

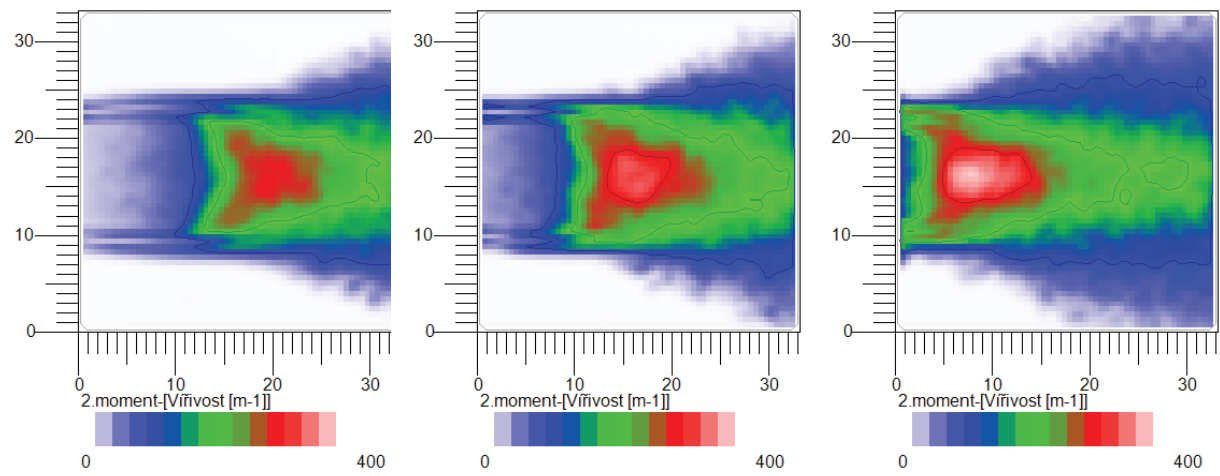

Fig. 9. Standard deviation of measured vorticity. The Reynolds numbers are from left: $2.55 \cdot 10^{3}$, $5.1 \cdot 10^{3}$ and $1.53 \cdot 10^{4}$.
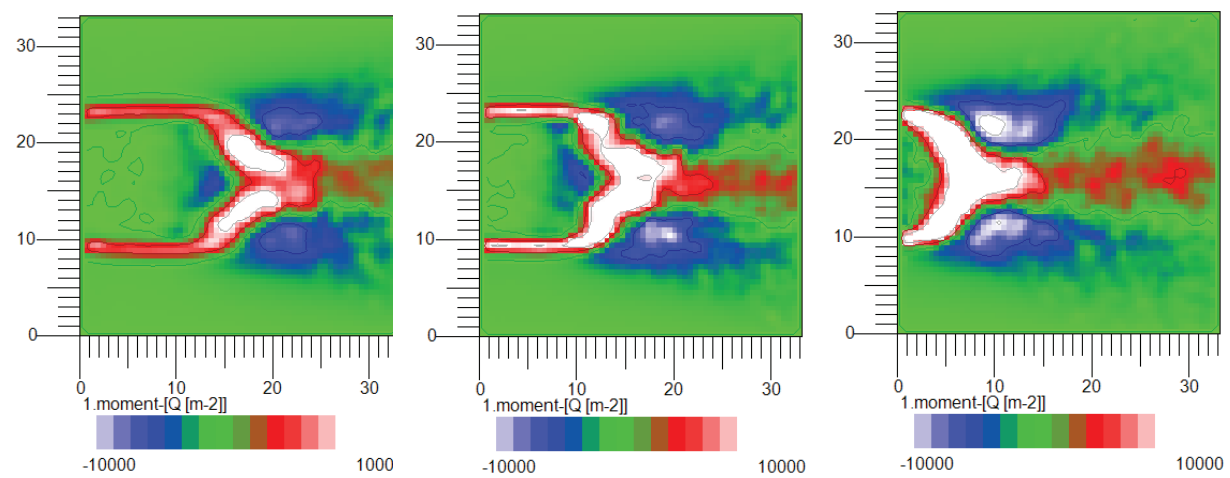

Fig. 10. Mean Q. The Reynolds numbers are from left: $2.55 \cdot 10^{3}, 5.1 \cdot 10^{3}$ and $1.53 \cdot 10^{4}$. Again, the velocity field is divided by the reference velocity, thus the unit of Q parameter changes from $\left[\mathrm{s}^{-2}\right]$ to $\left[\mathrm{m}^{-2}\right]$

Due to the nature of vorticity - it is made of two components rotation and shear - there apparently comes need to differentiate these two components of each other. Here we use the Q parameter

$$
Q=\frac{1}{2}\left(\omega^{2}-s^{2}\right)
$$

this physical quantity tells us which component of vorticity is predominant. Simplified, if Q is negative, the vorticity is made mainly of shear $s, s=\frac{1}{2}\left(\frac{\partial u_{i}}{\partial x_{j}}+\frac{\partial u_{j}}{\partial x_{i}}\right)$. If Q is positive, then component of rotation is predominant. The ensemble average of $\mathrm{Q}$ is displayed in Fig. 10 showing a single "Y-like" structure instead of pair of opposite branches as the vorticity do (Fig. 8). This is caused by the presence of vortices around the stagnation points, but these vortices are positive and negative averaging to zero signal, while the $\mathrm{Q}$ ensures summing, if the structure is rotational.

\section{Conclusion}

We used the experimental method of Particle Image Velocimetry (PIV) for measuring the instantaneous velocity past a circular cylinder [36] of diameter $10 \mathrm{~mm}$ placed inside an open wind tunnel test section. We observed a significant shortening of the recirculation bubble 
appearing in the ensemble-averaged maps. The fluctuations differ between components - the stream-wise component fluctuates most past the boundary layers of the cylinder with a local minimum at the wake axis, while the fluctuation of span-wise velocity has maximum at the axis.

In future we will continue analyzing this data, in focus to the individual vortices and their statistics, we plan to compare this results to some numerical simulation and later we will plan better next measurement learning from the current troubles and inaccuracies.

I gratefully thank to RNDr. Daniel Duda for supervising this small student project, although the measurement was limited due to the general limitations of student works at the university due to the COVID-19 pandemic. I gratefully thank to Ing. Martin Pelikán for the opportunity to attend the conference and for paying all the conference fees. I thank to Ing. Martin Novák for the organizing the student project selecting very interesting topics.

\section{References}

1. S.B. Pope, Turbulent Flows, Cambridge University Press, (2000)

2. G. Ilieva "A deep insight to secondary flows", Defect and Diffusion Forum 379: 83107 (2017)

3. D. Duda, T. Jelínek, P. Milčák, M. Němec, V. Uruba, V. Yanovych, P. Žitek, "Experimental Investigation of the Unsteady Stator/Rotor Wake Characteristics Downstream of an Axial Air Turbine”, Int. J. Turbomach. Propuls. Power 6(3): 22 (2021), doi: 10.3390/ijtpp6030022

4. J. Schottler, J. Bartl, F. Mühle, L. Sætran, J. Peinke, M. Hölling, "Wind tunnel experiments on wind turbine wakes in yaw: redefining the wake width", Wind Energ. Sci. 3: 257 - 273 (2018), doi: 10.5194/wes-3-257-2018

5. J. J. Niemela, L. Skrbek, K. R. Sreenivasan, "The wind in confined thermal convection”, J. Fluid Mech. 449: 169 - 173 (2001), doi: 10.1017/S0022112001006310

6. D. Duda, M. La Mantia, M. Rotter, L. Skrbek, "On the visualization of thermal counterflow of He II past a circular cylinder", J. Low Temp. Phys. 175: 331 - 338 (2014), doi: 10.1007/s10909-013-0961-z

7. L. Skrbek, P. Urban "Has the ultimate state of turbulent thermal convection been observed?" J. Fluid Mech. 785: 270 - 282 (2015), doi: 10.1017/jfm.2015.638

8. V. D. Arp, R. D. McCarty, D. G. Friend "Thermophysical properties of helium-4 from 0.8 to $1500 \mathrm{~K}$ with pressures to $2000 \mathrm{MPa"}$ " NIST technical note 1334 (1998)

9. C. H. K. Williamson, "Vortex Dynamics in the Cylinder Wake", Annual Review of Fluid Mechanics 28:1, 477-539 (1996), doi: 10.1146/annurev.fl.28.010196.002401

10. V. Strouhal, "Über eine besondere Art der Tonerregung”, Ann. Phys, 241: 216 - 251 (1878), doi: 10.1002/andp.18782411005

11. D. Duda, P. Švančara, M. La Mantia, M. Rotter, L. Skrbek "Visualization of viscous and quantum flows of liquid $\mathrm{He} 4$ due to an oscillating cylinder of rectangular cross section", Phys. Rev. B 92064519 (2015), doi: 10.1103/PhysRevB.92.064519

12. D. Duda, M. La Mantia, L. Skrbek "Streaming flow due to a quartz tuning fork oscillating in normal and superfluid He 4" Phys. Rev. B 92: 024519 (2017), doi: 10.1103/PhysRevB.96.024519

13. V. Yanovych, D. Duda, V. Uruba "Structure turbulent flow behind a square cylinder with an angle of incidence" European Journal of Mechanics B/Fluids 85: 110 - 123 (2021), doi: 10.1016/j.euromechflu.2020.09.003 
14. D. Duda "How Manufacturing Inaccuracies Affect Vortices in an Airfoil Wake" Proceedings Topical Problems of Fluid Mechanics 2021: 48 - 55 (2021), doi: 10.14311/TPFM.2021.007

15. W. Terra, A. Sciacchitano, F. Scarano, Aerodynamic drag of a transiting sphere by large-scale tomographic-PIV, Exp Fluids 58:83 (2017), doi: 10.1007/s00348-0172331-0

16. D. Duda "The PIV study of air flow past the counter-swirler 53983" MATEC Web of Conferences 168: 05004 (2018), doi: 10.1051/matecconf/201816805004

17. C. Tropea, A. L. Yarin, and J. F. Foss, "Springer handbook of experimental fluid mechanics", Springer (2007)

18. D. Duda, V. Uruba "PIV of air flow over a step and discussion of fluctuation decompositions" AIP Conference Proceedings 2000: 020005 (2018), doi: $10.1063 / 1.5049912$

19. D. Duda and V. Uruba, "Spatial spectrum form Particle Image Velocimetry data", ASME J of Nuclear Rad Sci. 5 (3): 030912 (2019)

20. C. H. K. Williamson, Three-dimensional wake transition. Journal of Fluid Mechanics, 328: 345-407. (2006), doi:10.1017/S0022112096008750

21. P. Pavlíček "Local Reynolds number” AIP Conference Proceedings 2118, 030035 (2019); doi: 10.1063/1.5114763

22. I. K. Marchevsky, K. S. Kuzmina, E. P. Ryatina "Flow Simulation around a Circular Cylinder for Low Reynolds Numbers and Determining the Flow Separation Point in VM2D Code" Proceedings Topical Problems of Fluid Mechanics 2020: 144 - 151 (2020), doi: 10.14311/TPFM.2020.019

23. C. H. K. Williamson "Vortex dynamics in the wake of a cylinder", chapter in S. I. Green "Fluid vortices", 155 - 234, Kluwer Academic Publishers (1995), isbn: 0-79233376-4

24. C. H. K. Williamson, A. Roshko "Measurement of base pressure in the wake of a cylinder at low Reynolds numbers" Zeitschrift für Flugwissenschaften und Weltraumforschung 14: 38 - 46 (1990)

25. C. Norberg "Effect of Reynolds numbers and a low-intensity freestream turbulence on the flow around a circular cylinder" Chalmers University of Technology, publication Nr. 87/2, S-412-96 (1987)

26. P. W. Bearman "On the vortex shedding from a circular cylinder in the critical Reynolds number regime" J. Fluid Mech. 37: 577 - 585 (1969), doi: $10.1017 / \mathrm{S} 0022112069000735$

27. W. C. L. Shih, C. Wang, D. Coles, A. Roshko "Experiments on flow past rough circular cylinders at large Reynolds numbers" ${ }^{\text {nd }}$ Int. Coll. On Bluff Body Aerod. And Applic., Melbourne (1992)

28. S. C. R. Dennis, G. Z. Chang "Numerical solutions for steady flow past a circular cylinder at Reynolds numbers up to 100" J. Fluid Mech. 42: 471 - 489 (1970), doi: $10.1017 / \mathrm{S} 0022112070001428$

29. V. Yanovych, D. Duda, V. Horáček, V. Uruba "Research of a wind tunnel parameters by means of cross-section analysis of air flow profiles" AIP Conference Proceedings 2189: 020024 (2019), doi: 10.1063/1.5138636

30. V. Yanovych, D. Duda "Structural deformation of a running wind tunnel measured by optical scanning" Strojnicky Casopis 70(2): 181 - 196 (2020), doi: 10.2478/scjme2020-0030 
31. D. Duda, T. Jelínek, M. Němec, V. Uruba, V. Yanovych, P. Žitek "Observation of flow structure past a full-stage axial air turbine at the nominal and off-design states" AIP Conference Proceedings 2323: 030004 (2020), doi: 10.1063/5.0041491

32. D. Duda, T. Jelínek, M. Němec, V. Uruba, V. Yanovych, P. Žitek "Particle image velocimetry measurement inside axial air test turbine - Effect of window" AIP Conference Proceedings 2323: 030005 (2020), doi: 10.1063/5.0041492

33. D. Duda, J. Bém, V. Yanovych, P. Pavlíček, V. Uruba "Secondary flow of second kind in a short channel observed by PIV" European Journal of Mechanics B/ Fluids 79: 444 - 453 (2020), doi: 10.1016/j.euromechflu.2019.10.005

34. D. Duda, V. Yanovych, V. Uruba "An experimental study of turbulent mixing in channel flow past a grid" Processes 8: 1 - 17 (2020), doi: 10.3390/pr8111355

35. D. Duda "Preliminary PIV measurement of an air jet" AIP Conference Proceedings 2047: 020001 (2018), doi: 10.1063/1.5081634

36. V. Uruba, P. Procházka, "The Reynolds number effect on dynamics of the wake behind a circular cylinder", AIP Conference Proceedings 2189, 020023 (2019), doi:

$10.1063 / 1.5138635$ 